\title{
Design and Implementation of the Electric Propulsion Train of a Prototype for the Eco Shell Marathon Competition
}

\section{Diseño y Implementación del Tren de Propul- sión Eléctrico de un Prototipo para la Compe- tencia Eco Shell Marathon}

\author{
J. Pancha, J. Néjer, V. Romero, and J. Morocho
}

VII International Congress of

Escuela Superior Politécnica de Chimborazo, Riobamba, Ecuador

Science, Technology,

Entrepreneurship and

Innovation (SECTEI 2020)

Corresponding Author:

V. Romero

Vicente.romero@espoch.edu.ec

Published: 26 August 2021

Production and Hosting by

Knowledge $\mathrm{E}$

(c) J. Pancha et al. This article is distributed under the terms of the Creative Commons Attribution License, which permits unrestricted use and redistribution provided that the original author and source are credited.

\section{Abstract}

The main objective of this thesis project focused on designing and implementing an electric propulsion train for a vehicle prototype and subsequently achieving an adequate driving technique, where man and machine come together to achieve an efficient participation in the Eco Shell Marathon competition. In this context, the methodology sought to identify needs such as: equipment and driving strategies. Therefore, for the equipment selection, the technique of the ordinal method corrected of weighted criteria was applied, resulting in: a Brushed Speed WPHMOTO 48v 1000w motor with a score of percent; A TDPRO YK31C Accelerator Driver with a 40 percent priority, and Turnigy Graphene Panther Batteries $(6000 \mathrm{mAh}, 6 \mathrm{~S} 75 \mathrm{C}$ Lipo with XT90) with a 44 percent priority, from three possible options for each of them. After the equipment implementation, experimental trials of four types of practices were carried out. In the analysis of the results when contrasting the first two practices carried out on the sloping track zero degrees, it is revealed that in Test 01 the vehicle travels $35.056 \mathrm{~km}$ per $\mathrm{kW} . \mathrm{h}$, while in Test 02 the distance is $37.466 \mathrm{~km}$ per each kW.h. In the case of Test 03 and 04 performed on the track with an inclination of two degrees, the coefficients of the relationship between distance traveled and energy consumed are: $34.29 \mathrm{~km} / \mathrm{kWh}$ and $42.219 \mathrm{~km} / \mathrm{kW} . \mathrm{h}$ respectively. Therefore, the relationship between the distance traveled and energy consumed present favorable data when applying the driving strategy based on giving impulses through the accelerator as the speed of the two types of tracks decreases.

Keywords: technology and engineering sciences, electric vehicle, electric propulsion train, energy efficiency, energy management, energy efficient race, lithium ion batteries.

\section{Resumen}

En este presente proyecto de tesis el objetivo principal se fundamentó en diseñar e implementar un tren de propulsión eléctrico para un prototipo de vehículo y posteriormente conseguir una técnica adecuada de conducción, donde hombre y maquina se junten para lograr una participación eficiente en la competencia Eco Shell Marathon. Bajo este contexto la metodología se regio al reconocimiento de necesidades como: equipos y estrategias de conducción. Por tanto, para la selección de equipos se aplicó la técnica de método ordinal corregido de criterios ponderados, dando como resultados: un motor Brushed Speed WPHMOTO 48v 1000w, con una puntuación de treinta y seis por ciento; Un Driver Acelerador TDPRO YK31C, con una prioridad de cuarenta por ciento y Baterías Turnigy Graphene Panther (6000mAh, 6S 75C Lipo con XT90), con una prioridad de cuarenta y cuatro por ciento, de 
tres posibles opciones para cada uno de ellos. Posterior a la implementación de equipos, se procede a las practicas experimentales de cuatro tipos de ensayos. En cuyo análisis de resultados al contrastar los dos primeros ensayos realizados sobre la pista inclinada cero grados, revela que el Ensayo 01 el vehículo recorre 35,056 km por cada kW.h, mientras que en el Ensayo 02 el recorrido es de 37,466 km por cada kW.h. Para el caso del Ensayo 03 y 04 , realizados sobre la pista con una inclinación de dos grados, los coeficientes de la relación entre distancia recorrida y energía consumida son: 34,279 km/kW.h y 42,219 km/kW.h respectivamente. En tal virtud la relación distancia recorrida y energía consumida presenta datos favorables al aplicar la estrategia de conducción basada en dar impulsos mediante e acelerador al disminuir la velocidad en los dos tipos de pistas.

Palabras Clave: vehículo eléctrico, tren de propulsión eléctrico, eficiencia energética, baterías de iones de Litio.

\section{Introducción}

En la actualidad las fuentes de energía tradicionales se vuelven cada día más limitadas y las normativas de impacto ambiental demandan un cambio en el modelo de consumo energético, perspectivas que apenas inician por una sociedad que luchan por proporcionar una calidad de aire aceptable y niveles de ruido para sus habitantes.

Estudios acerca de la contaminación del aire ambiental han revelado datos aproximados de 3,7 millones de muertes prematuras anualmente en todo el mundo y la contaminación del aire por las emisiones del transporte terrestre, plantea un problema significativo en las ciudades [1].

La contaminación atmosférica provocada por los vehículos automotores constituyen la causa principal del deterioro de la calidad del aire del medio ambiente en muchos centros urbanos de América Latina [2].

Atendiendo estas consideraciones y desde el punto de vista de la Ingeniería Automotriz se pone en la mira el avance tecnológico de la movilidad y el transporte, teniendo como enfoque primordial mejorar o desacoplar la demanda de transporte relacionada con el consumo de combustible fósil.

Por lo que en la actualidad se hace presente el desarrollo de diseño de vehículos eléctricos, mismos que presenta algunos parámetros por investigar para mejorar su rendimiento, entre ellos el de tren motriz, la implementación de componentes y la estrategia de gestión de energía.

Bajo este contexto existen compañías que impulsan a la comunidad investigativa mediante apoyo financiero y/o socializan a través de concursos, una de ellas la competencia Shell EcoMarathon. 
El Shell Eco-Marathon es una competencia patrocinada por Shell para equipos de estudiantes de escuelas secundarias y universidades de todo el mundo, la cual se lleva acabo anualmente, los equipos participantes construyen vehículos especiales para lograr la mayor eficiencia energética posible [3].

La Escuela Superior Politécnica de Chimborazo con el equipo ECO Kuntur ESPOCH conformado por estudiantes y docentes de la Facultad de Mecánica asumió el reto de participar en la competencia Shell Eco-Marathon 2018 y 2019, donde demostraron destrezas de ingeniería y el compromiso con la institución para lograr aprobar las exigentes inspecciones técnicas y de seguridad del prototipo y tener acceso a pista con un prototipo.

Con estos antecedentes, ECO KUNTUR se plantea dejar en alto este año en la participación en el circuito de Sonoma, California Estados Unidos 2020.

A través de la ejecución de este proyecto, donde se realizará el diseño del tren motriz eléctrico, la configuración de los componentes mediante la implementación y posteriormente conseguir una técnica adecuada de conducción que no es otra cosa que inmiscuirse en la estrategia de gestión, con un solo propósito, el de conseguir un bajo consumo de energía y la preservación del estado de la batería.

\subsection{Consideraciones teóricas}

\subsubsection{Sistema de propulsión}

El sistema de propulsión o tren motriz de un vehículo convencional como el de un vehículo eléctrico es el mismo para moverse, es decir transmiten energía mecánica a las ruedas y como resultado da el desplazamiento del vehículo [4] (p. 9).

El tren motriz de cualquier vehículo es el conjunto mecánico que permite la propulsión del vehículo y está constituido por el motor y transmisión [5] (p. 22).

\subsubsection{Vehículo eléctrico}

Un vehículo eléctrico para moverse tanto parcial como totalmente maneja un motor eléctrico provisto con baterías recargables por corriente. La energía química de la batería se transformará en energía eléctrica para posteriormente convertirse en energía mecánica por medio de un motor eléctrico [4] (p. 10, 11).

\section{Metodología}




\subsection{Procedimiento de diseño}

Para el desarrollo de la presente investigación, se efectuará las siguientes actividades de manera ordenadas y en un número finito de procesos, las cuales se describen en el siguiente flujograma de la Figura 1.

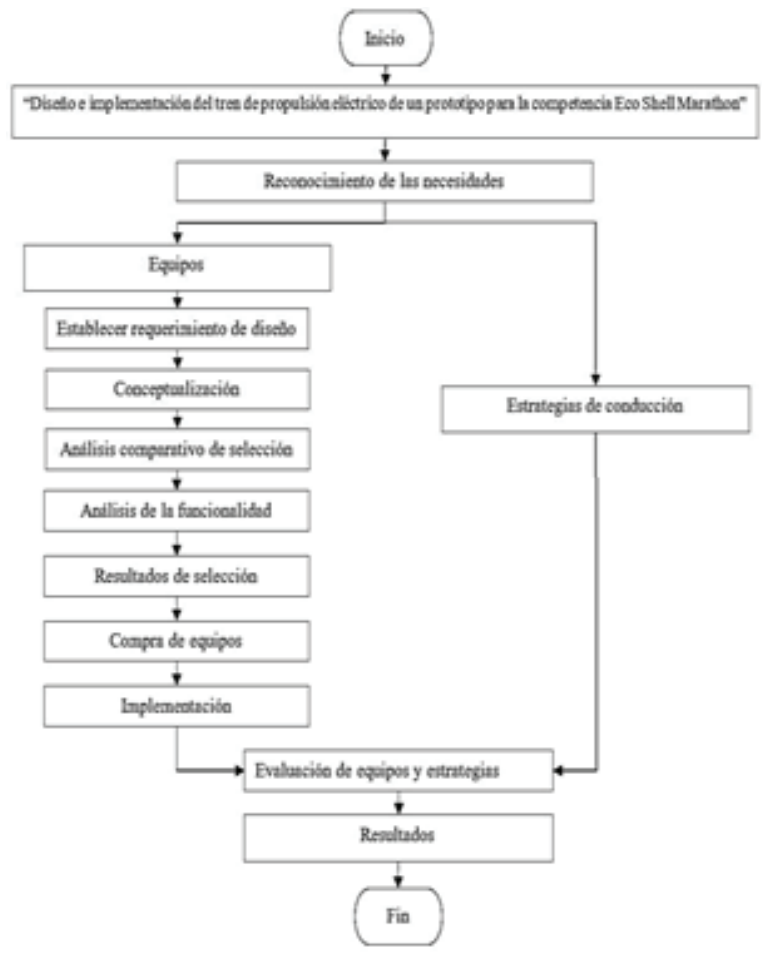

Figure 1

Flujograma del procedimiento.

\subsection{Reconocimiento de las Necesidades}

En este estudio la necesidad es implementar un tren de propulsión eléctrico de un prototipo para reducir el consumo de energía en una batería, mediante una selección ingenieril de equipos y técnicas de conducción, que desemboquen en ahorro de energía.

\subsubsection{Equipos}

En cuanto a equipos se requiere adquirir un motor eléctrico, su controlador y baterías. Mismos que deben satisfacer los requerimientos del reglamento de competencia y a su vez posibiliten un buen desempeño del vehículo en la pista. 


\section{Análisis comparativo de selección}

Para la selección del motor eléctrico, controlador y batería se aplicará una matriz del método ordinal corregido de criterios ponderados, lo cual permitirá obtener resultados globales suficientemente significativos, en los tres equipos como se muestra:

\section{Motor}

En la selección del motor se tiene tres motores eléctricos los cuales se menciona a continuación:

- Motor A: Turnigy Rotomax $100 \mathrm{cc}$ Tamaño $167 \mathrm{kv}$ sin escobillas del motor Outrunner. Motor B: Brushed Speed motor WPHMOTO 48v 1000W.

- Motor C: Motor de scooter eléctrico 48v 1000W.

La Tabla 1 Muestra las puntuaciones de los tres motores eléctricos, para determinar cuál de ellos tiene la de mayor prioridad.

\section{Table 1}

Tabla de conclusión de prioridad del motor.

\begin{tabular}{lll|l|l|l|l|l|} 
& Pote n-cia & Efici encia & Velo cidad & Peso & Precio & $\boldsymbol{\Sigma}$ & Pri oridad \\
Mo- tor A & 0,17 & 0,04 & 0,10 & 0,07 & 0,01 & 0,39 & 1 \\
Mo- tor B & 0,08 & 0,13 & 0,07 & 0,04 & 0,03 & 0,36 & 2 \\
Mo- tor C & 0,09 & 0,09 & 0,03 & 0,02 & 0,02 & 0,25 & 3
\end{tabular}

Fuente: Autores.

Realizado por: Autores.

El Motor A, está mejor puntuado, pero por funcionalidad entre motor y el controlador esta opción no es viable, en tal virtud se seleccionó la segunda alternativa Motor B: Brushed Speed motor WPHMOTO 48v 1000W, con una puntuación 36\%, cuyos datos técnicos se muestran en la Tabla 2.

\subsubsection{El controlador}

Para el caso del controlador se tiene tres ejemplares los cuales se menciona a continuación:

- Controlador A: Driver Acelerador TDPRO YK31C

- Controlador B: Driver Acelerador 45A-24 MOSFET

- Controlador C: Driver Acelerador 33-15 MOSFET 


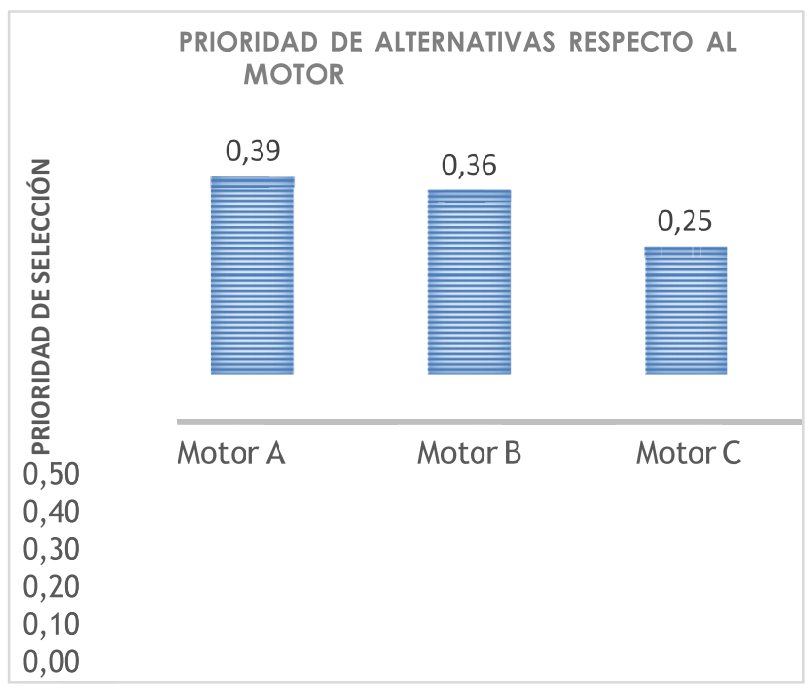

Figure 2

Conclusión de la prioridad de alternativas con respecto al motor.

Table 2

Datos técnicos del Brushed Speed motor WPHMOTO 48v 1000W.

\begin{tabular}{l|l} 
Motor & Brushed Speed motor WPHMOTO 48v 1000W \\
\hline Potencia de salida & $1000 \mathrm{~W}-1,4 \mathrm{HP}$ \\
\hline Velocidad nominal & $3000-3360 \mathrm{RPM}$ \\
\hline Tensión nominal & $48 \mathrm{~V} \mathrm{DC}$ \\
\hline Corriente Clasificada & $27 \mathrm{~A}$ \\
\hline Corriente sin carga & $\leq 2,8 \mathrm{~A}$ \\
\hline Eficiencia máxima a 48 V & $80 \%$ \\
\hline Piñón & 9 dientes para cadena T8F de $8 \mathrm{~mm}$ \\
\hline
\end{tabular}

Fuente: Referencia [6].

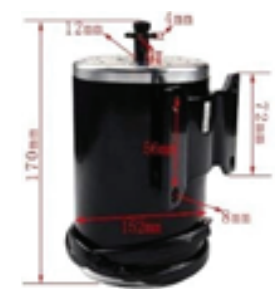

Figure 3

Brushed Speed motor WPHMOTO 48v 1000W.

Luego de la evaluación de los pesos específicos de las distintas soluciones para cada criterio la Tabla 3, muestra la prioridad uno el Controlador A. 
Table 3

Tabla de conclusión de prioridad del controlador.

\begin{tabular}{l|l|l|l|l|l|l|} 
& Peso & Tensión & Efi. & Precio & & Priod. \\
Cont. A & 0,2 & 0,05 & 0,10 & 0,05 & 0,4 & 1 \\
Cont. B & 0,14 & 0,13 & 0,03 & 0,03 & 0,33 & 2 \\
Cont. C & 0,07 & 0,12 & 0,07 & 0,02 & 0,28 & 3
\end{tabular}

Fuente: Autores.

Realizado por: Autores.

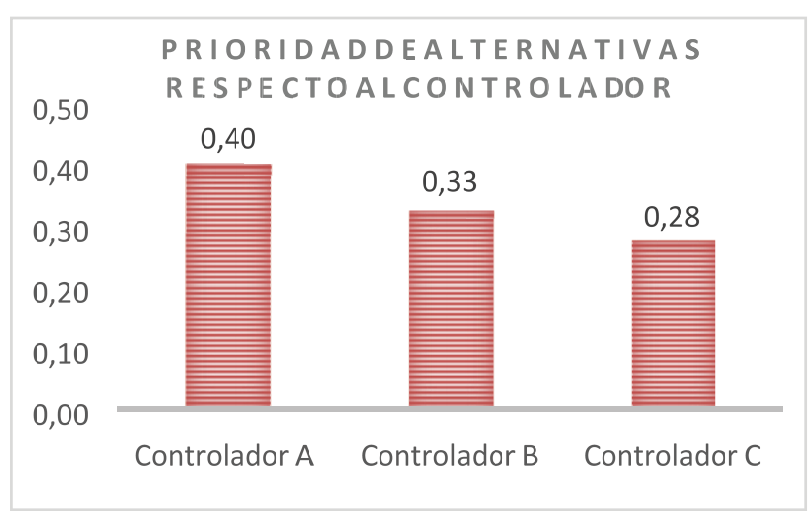

Figure 4

Conclusión de la prioridad alternativas de controlador.

De tres controladores el de mejor puntuación es el Controlador A: Driver Acelerador TDPRO YK31C, con una puntuación del 40\%. En la Tabla 4, se muestras los datos técnicos.

\section{Table 4}

Datos técnicos del controlador.

\begin{tabular}{ll} 
Controlador & Driver Acelerador TDPRO YK31C \\
\hline Tensión nominal & $1000 \mathrm{~W}-1,4 \mathrm{HP}$ \\
\hline Motor de partida & $48 \mathrm{v} 1000 \mathrm{~W}$ \\
\hline Eficiencia de conversión & $95 \%$ \\
\hline Protección de bajo voltaje & $42 \pm 0,5 \mathrm{v}$ \\
\hline Regulador de velocidad (acelerador) & $1-4 \mathrm{v}$ \\
\hline
\end{tabular}

Fuente: Referencia [6].

\subsubsection{La batería}

De igual forma para la selección de baterías se tiene de igual forma tres presentaciones, las cuales se menciona a continuación: 


\section{Figure 5}

Driver Acelerador TDPRO YK31C.

- Baterías A: Kit de motor 48v 1000W 45A

- Turnigy Graphene Panther (6000mAh, 6S 75C Lipo con XT90)

- Baterías C: Kit de motor 45V-33a

Finalmente se tiene la Tabla 5, de conclusiones de del método ordinal corregido de criterios ponderados, para indicar la prioridad de estos equipos.

\section{Table 5}

Tabla de conclusión de prioridad de las baterías.

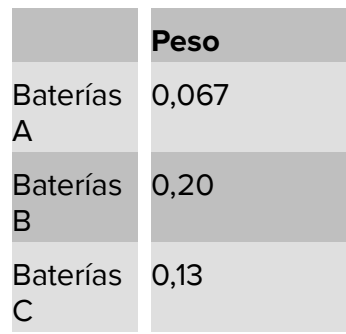

Volt.
0,042
0,10
0,11

Desg. Const.
0,125
0,09
0,04

Precio
0,017
0,05
0,03

\begin{tabular}{l|l} 
& Prid. \\
0,25 & 3 \\
0,44 & 1 \\
0,31 & 2
\end{tabular}

Fuente: Autores

Realizado por: Autores.

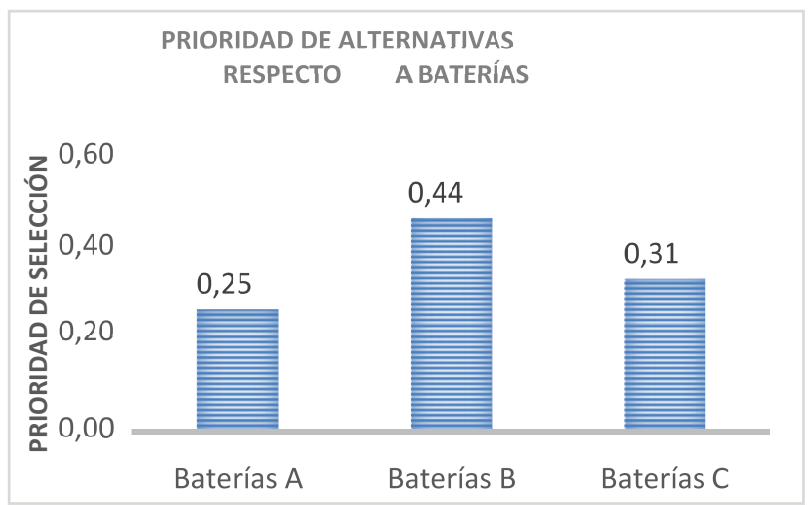

Figure 6

Turnigy Heavy Duty 5000mAh 7S 60C Lipo Pack w/XT90.

Mediante el análisis de selección la tabla de conclusiones da como resultado la prioridad las Baterías B: Turnigy Graphene Panther (6000mAh, 6S 75C Lipo con XT90), con una puntuación de $44 \%$ de prioridad. En la Tabla 6 se muestra las sus especificaciones técnicas. 
Table 6

Datos técnicos del paquete de Baterías Turnigy Graphene Panther (6000mAh, 6S 75C Lipo con XT90).

Paquete de Baterías
Capacidad
Recuento de celdas
Voltaje
Descarga constante
Descarga máxima (3s)
Peso

Turnigy Graphene Panther (6000mAh, 6S 75C
Lipo con XT90)
$6000 \mathrm{mAh}$
6
$22,2 \mathrm{v}$
$75 \mathrm{C}$
$150 \mathrm{C}$
$1,140 \mathrm{~kg}$

Fuente: Referencia [6]

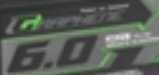

Figure 7

Turnigy Graphene Panther (6000mAh, 6S 75C Lipo con XT90).

\subsubsection{Implantación}

Una vez adquirido los equipos seleccionados, se realiza la implementación de los mismos al prototipo, para lo cual es fundamental presentar los diagramas mecánico y eléctrico, para interpretar su ensamblaje.

\section{Diagrama mecánico del sistema}

Es relevante mostrar un esquema o diagrama mecánico de cómo esta acoplado y el motor y la rueda dentada motriz y cuál es la relación de transmisión para posteriores cálculos en la obtención de velocidades de entrada y salida, datos importantes para la obtención de la eficiencia.

\section{Diagrama eléctrico}

Otra tarea prioritaria fue la instalación eléctrica rigiéndose minuciosamente al diagrama eléctrico y al reglamento, que exige dotar al vehículo medidas de seguridad para evitar accidentes en la pista o zona de paddocks. 


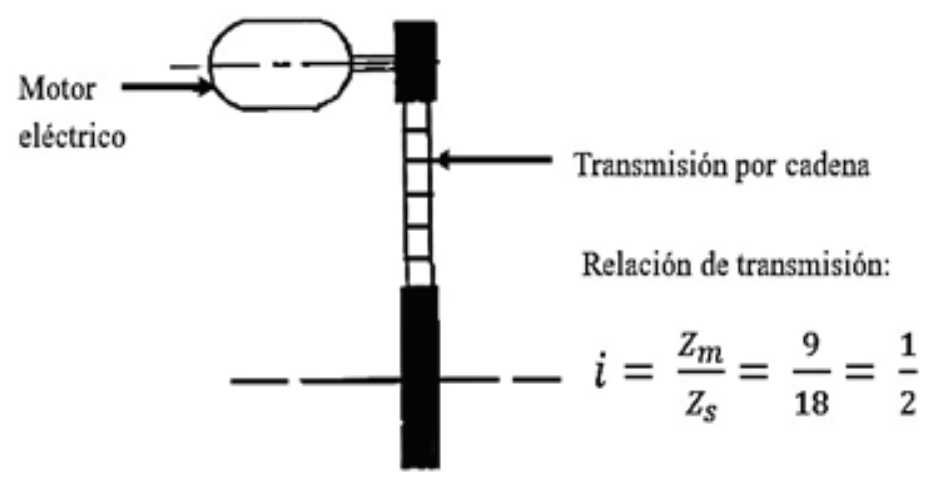

Figure 8

Diagrama mecánico.

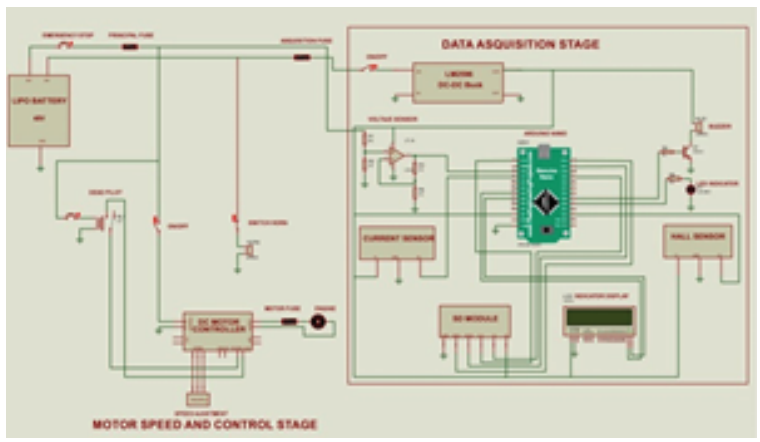

Figure 9

Diagrama eléctrico.

\subsubsection{Estrategias de conducción}

Para el caso de las metodologías de conducción se ha optado por estudiar dos estrategias para cada condición de pista. A continuación, se asigna una nomenclatura y de especifica detalles de las pistas y técnicas de conducción.

\section{Pista}

- PISTA A.- Inclinación de la pista $0^{\circ}$.

- PISTA B.- Inclinación de la pista $2^{\circ}$.

\section{Metodologías}

- Estrategia 01: Aceleración máxima, pisando el acelerador de manera permanente.

- Estrategia 02: Velocidad ideal constante, dando impulsos mediante el acelerador solo cuando disminuya la velocidad, tratando de conseguir siempre una velocidad constante, al momento de arrancar iniciar en la 5ta marcha para disminuir el 
consumo de amperaje debido a que en este momento es cuando existe el mayor consumo de tensión, a medida que va avanzando el prototipo para tener mayor velocidad regresar al 1er cambio.

\subsection{Evaluación de Equipos y estrategias}

Una vez realizado el diseño e implementación se procede a la experimentación de las metodologías de conducción, planteando las necesidades de competencia.

\subsubsection{Recolección de datos}

Mediante la utilización de instrumentos se procedió a realizar la medición de variables como: Tiempo, voltaje, intensidad de corriente, velocidad, potencia y distancia recorrida del vehículo, los mismos que son indispensables para el análisis cuantitativo de resultados.

Los datos de campo con los cuales se trabajó para la evaluación de la estrategia, corresponden a valores promedios de cada una de las variables de diez practicas realizadas por cada ensayo

\subsubsection{Análisis de resultados}

\section{Análisis de la estrategia}

Para la determinación de la mejor metodología se desarrollará un análisis comparativo, como indica la Figura 7, entre las dos estrategias planteadas en esta investigación, bajo las condiciones de Pista A y Pista B, de esta forma se puede apreciar cuál de estas es la más adecuada en cada tipo de pista.

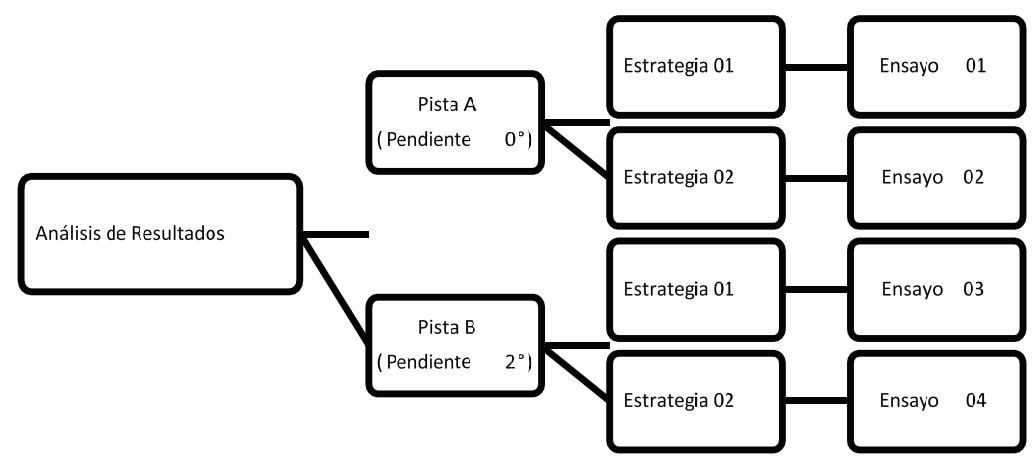

Figure 10

Diagrama del análisis de resultados. 


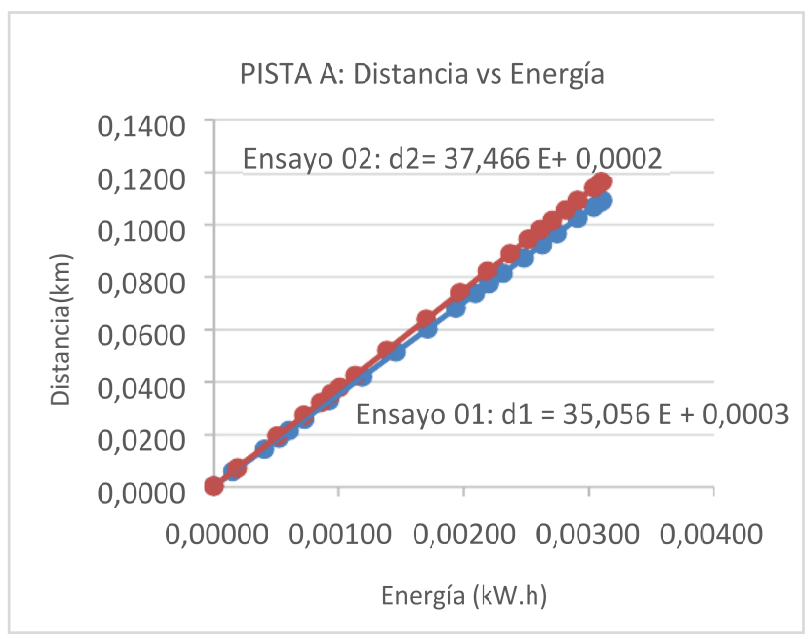

Figure 11

Comparación de Estrategia 01 y estrategia 02 de conducción en la Pista A

PISTA B: Distancia vs Energía

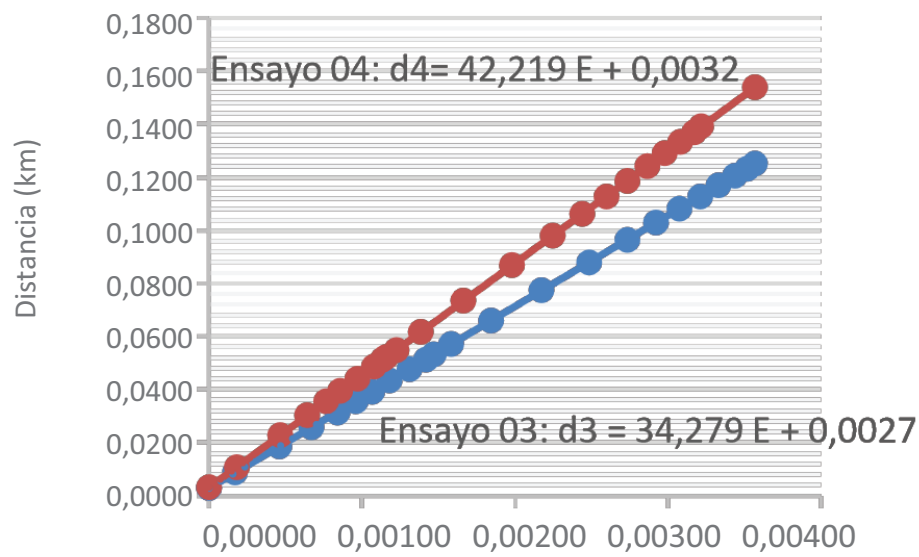

Figure 12

Comparación de Estrategia 01 y estrategia 02 de conducción en la Pista B.

- Contrastación de las Gráficas Distancia vs Energía, para apreciar la diferencia de las pendientes de las dos curvas linealizadas de datos experimentales obtenidos en el ensayo 01 y 02. Donde las Gráficas 4 y 5 indican una pendiente superior correspondiente a las curvas generadas por la Estrategia 02 con respecto a la Estrategia 01 en las dos pistas.

- Comparación cuantitativa de la tasa de variación de la distancia recorrida en (km) por el vehículo y la energía consumida por cada (kw.h) correspondiente al valor de las pendientes de las curvas linealizadas. La Grafica 6 Muestra la superioridad de la variación de la distancia medida en kilómetros por cada kw.h de energía 


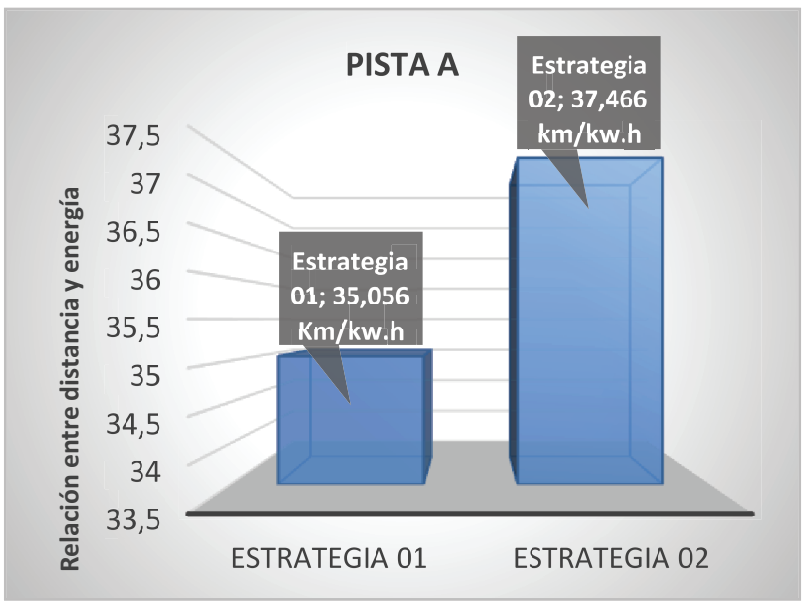

Figure 13

Comparación de la relación distancia con la energía en [km/kW.h] de las estrategias de conducción en la Pista A.

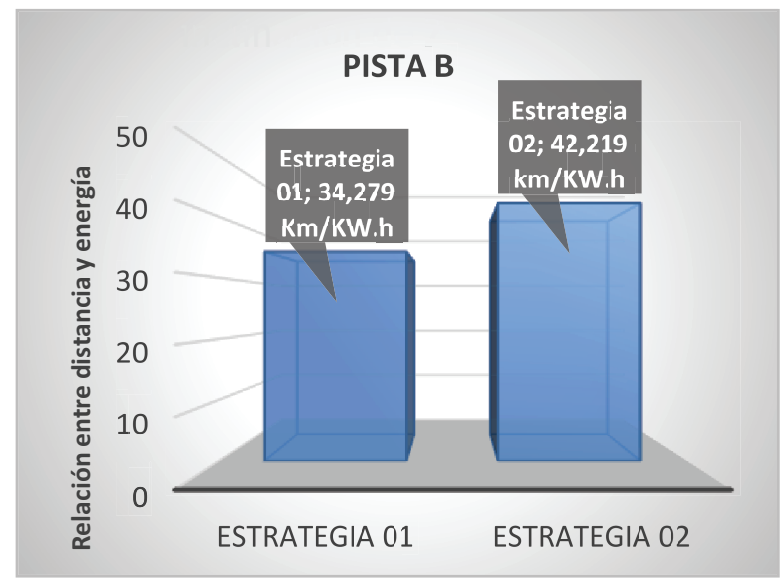

\section{Figure 14}

Comparación de la relación distancia con la energía en [km/kW.h] de las estrategias de conducción en la Pista $B$.

consumida de la fuente en la estrategia 02 en una pista con una inclinación de $0^{\circ}$.

La Gráfica 7 Muestra la superioridad de la variación de la distancia medida en kilómetros por cada kw.h de energía consumida de la fuente en la estrategia 02 en una pista con una inclinación de $2^{\circ}$.

- Comparación cuantitativa de la energía consumida en 6,580 km, correspondiente a la distancia establecida por la competencia eco Shell Eco Marathon.

La Gráfica 8 Muestra a la estrategia 01 consumir mayor energía con respecto a la estrategia 02, para un mismo recorrido, por ende, se puede concluir que la estrategia 02 en la pista de inclinación $0^{\circ}$ es más eficiente. 


\section{PISTA A}

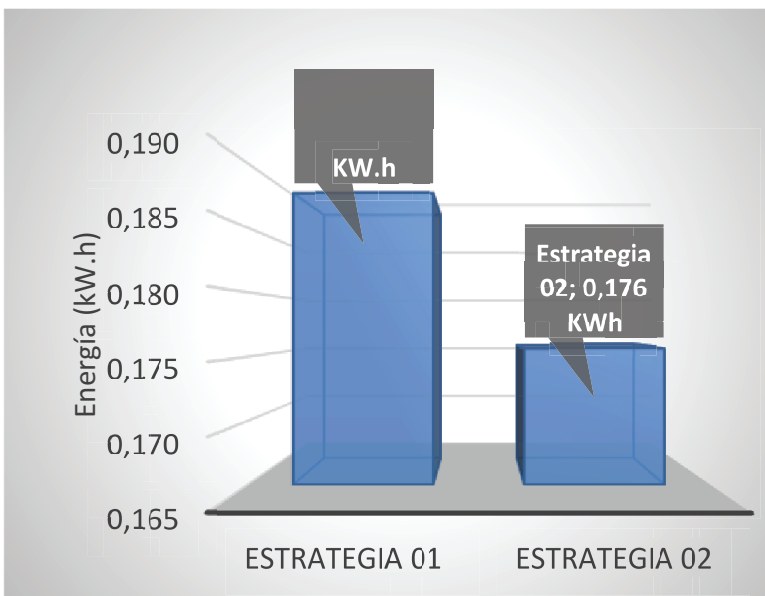

Figure 15

Comparación de energía consumida en 6,584 km en las estrategias de conducción en la Pista A. Realizado por: Autores.

La Grafica 9 Muestra a la estrategia 02 consumir menor energía con respecto a la estrategia 01, para un mismo recorrido, por ende, se puede concluir que la estrategia 02 en la pista de inclinación $0^{\circ}$ es más eficiente.

\section{PISTA B}

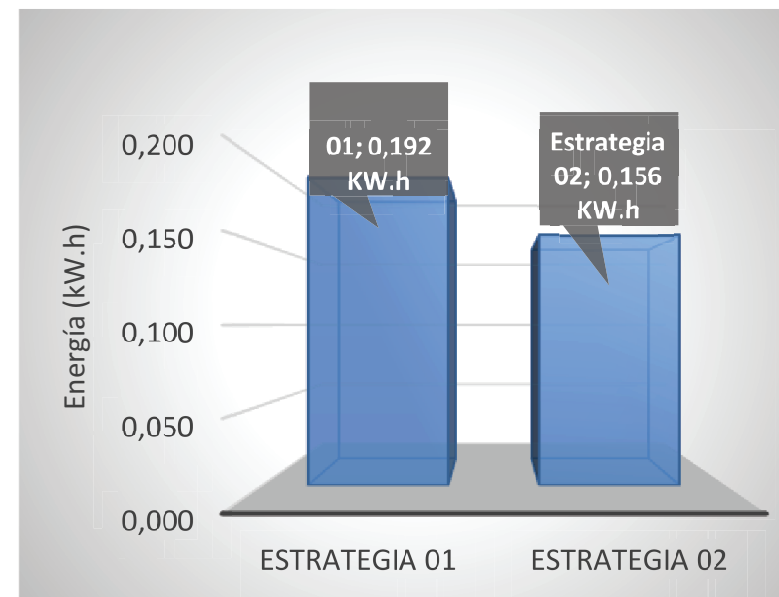

Figure 16

Comparación de energía consumida en 6,584 km en las estrategias de conducción en la Pista B. Realizado por: Autores. 


\section{Análisis de la eficiencia del prototipo de vehículo eléctrico}

Para el análisis de la eficiencia se tiene el recurso matemático $n=\frac{E_{s}}{E_{o}}$, por tal razón se requiere hallar la energía de salida en base a datos experimentales, donde mediante el recurso matemático concerniente a energía cinemática se calculó dicha energía que desarrolla el prototipo de vehículo eléctrico, como se presenta a continuación.

$$
E_{c}=\frac{1}{2} * m * v_{i} \text {. }
$$

Con el objetivo de conocer la eficiencia global del vehículo, en cualquier condición de la pista probada y cual sea la estrategia utilizada de las dos presentadas se halla una eficiencia energética promedio que caracteriza el trabajo de diseño e implementación de un tren de propulsión eléctrica igual a:

$$
n p=90,87 \% .
$$

\section{Discusión}

De los resultados obtenidos conllevan a sugerir que la metodología adecuada de conducción es la Estrategia 02, ya sea en la Pista A o en la Pista B, con una inclinación de $0^{\circ}$ y $2^{\circ}$ respectivamente, pues esta presenta una mayor tasa de variación de la distancia recorrida en [km] con respecto a la energía consumida en por cada [kW.h].

\section{Conclusiones}

El diseño del tren motriz eléctrico seleccionado mediante el método ordinal corregido de criterios ponderados; es un motor Brushed Speed motor WPHMOTO 48v 1000W, un Driver Acelerador TDPRO YK31C y Baterías Turnigy Graphene Panther (6000mAh, 6S 75C Lipo con XT90); y conjuntamente las estrategias de conducción cumplen el objetivo de conseguir un prototipo de vehículo eléctrico eficiente en un $91 \%$.

En la relación de transmisión se ha implementado un sistema accionado por cadena cuya relación de transmisión es $i=\frac{Z_{m}}{Z_{s}}=\frac{9}{18}=\frac{1}{2}$; relación con la cual se consigue un rendimiento del $96 \%$.

En tal virtud el vehículo tendrá un mínimo consumo del amperaje, máximo rendimiento en la rueda motriz, preservación del estado de la batería y alta esperanza de vida con un menor ruido de la cadena.

Se ha analizado el consumo de energía en los 6,580 km que debe recorrer el vehículo en la competencia, donde las prácticas experimentales conllevan a concluir que la técnica adecuada de conducción ya sea en la Pista $A$ o en la Pista $B$, con una inclinación de $0^{\circ}$ y $2^{\circ}$ respectivamente, es la estrategia 02 ya que presenta una mayor tasa de variación de la distancia recorrida en [km] con respecto a la energía consumida en 
[kW.h], es decir se considera optimo aplicar la Estrategia 02, misma que consiste en dar impulsos con el acelerador, a la percepción de disminución de velocidad que será determinada por el piloto.

\section{References}

[1] Brushless. 2016. http://motores.nichese.com/brushless.htm.

[2] Carreño E et al. Diseño y fabricación de un vehículo autónomo impulsado por energía solar. Dialnet. 2016. Available from: https://dialnet.unirioja.es/servlet/ articulo?codigo $=3957091$.

[3] Cebrian J. Mecánica y Entretenimiento Simple del Automóvil. Madrid: Ministerio Del Interior; 2016.

[4] Cuesta G. 2017. UPCommons. 2017 September 23. Available from: https:// upcommons.upc.edu/handle/2117/108997?show=full.

[5] Honda small engines. Honda. 2019. https://engines.honda.com/models/modeldetail/ mini-4.

[6] Ibañez. Xataka. 2017 July 31. Available from:https://www.xataka.com/automovil/ estosera-lo-proximo-en-baterias-para-cocheselectricos-mas-de-650-km-de-autonomia-real.

[7] Iglesias R et al. Scribd. 2019. Available from: https://es.scribd.com/document/266674134/ Modelado-y-Simulacion-de-Una-Bateria-de-IonLitio-Comercial-Multicelda-1.

[8] Izrurzun R, Garcia F. Los Coches Eléctricos. El Ecologista, 2015;21.

[9] Kim. Contaminación del aire ambiental. Paho. 2017. https://www.paho.org/hq/index. php?option=com_content\&view=article\&id=12918:ambient-airpollution\&ltemid= $72243 \&$ lang $=$ es.

[10] Nieto A. Elementos de máquina. 2017.

[11] Quenaya E. 2018. Impacto de la contaminación del aire por el parque automotor en la población del área urbana de Arequipa 2016 - 2017. Repositorio. 2018. http: //repositorio.unsa.edu.pe/handle/UNSA/796

[12] Rosero D, Lopez, E. Repositorio universidad técnica de ambato. 2018. http:// repositorio.uta.edu.ec/jspui/handle/123456789/27922.

[13] Shell. Shell Global. 2019. https://www.shell.com/make-the-future/shellecomarathon. html.

[14] Turnigy Graphene Panther. Baterias turnigy. 2019. [En línea] 2019. Available from: https://hobbyking.com/en_us/ turnigy-graphene6000mah-6s-75c-lipo-pack-w-xt90.html.

[15] Ugaulde H. 2015. Diseño de tren motriz para vehículo de competición en Shell Eco Marathon Americas. [En línea] 2015. [Citado el: 15 de Octubre de 2019.] http: //132.248.52.100:8080/xmlui/handle/132.248.52.100/5991. 